\title{
Empirical and theoretical limits on lag recency in free recall
}

\author{
Simon Farrell \\ Department of Experimental Psychology \\ University of Bristol \\ Stephan Lewandowsky \\ School of Psychology \\ University of Western Australia
}

\begin{abstract}
One widely accepted empirical regularity in free recall holds that when people successively transition from report of one list item to another, they prefer transitions across short lags (e.g., by reporting items from adjacent serial positions) to transitions involving large lags. This regularity has provided crucial support for the Temporal Context Model (TCM), a model of the evolution of temporal context in episodic memory (M. W. Howard \& M. J. Kahana, 2002). We report a re-analysis of 14 data sets which shows that contrary to the presumed preference for short lags, people often produce transitions with larger lags during recall. We show that these data cannot be accommodated by TCM. We furthermore show that existing applications of the model have, for mathematical convenience, introduced assumptions that circumvented its core principle of context evolution. When we instantiate TCM as verbally described, with a gradually evolving context, we find that its behavior qualitatively departs from the version currently implemented, and that the model is still unable to capture the nature of transitions in free recall. We conclude that TCM requires further modification and development before it can explain the data that constitute its main source of support. Supplementary material relevant to this article can be downloaded from www.psychonomic.org/archive.
\end{abstract}

The free recall task has been prominent in theorizing about episodic memory (e.g., Brown, Neath, \& Chater, 2007 ; Laming, 1999 ; Raaijmakers \& Shiffrin, 1981 ; Tan \& Ward, 2000; for an early review, see Murdock, 1974). A particularly intense debate surrounds the origin

Collaboration on this project was assisted by an Australian Research Council International Linkage Grant to Stephan Lewandowsky, Gordon Brown, and Simon Farrell. The second author was supported by an Australian Professorial Fellowship from the Australian Research Council. We thank Marc Howard for his clarification of some details of the operation of the model. Correspondence should be addressed to Simon Farrell, Department of Psychology, University of Bristol, 12a Priory Road, Clifton, Bristol BS8 1TU, UK; e-mail: Simon.Farrell@bristol.ac.uk. 
and nature of the recency effect, which refers to the greatly enhanced recall of items from the last few list positions (e.g., Postman \& Phillips, 1965). Recency has been variously ascribed to a limited-capacity short-term buffer (Glanzer \& Cunitz, 1966 ; Postman \& Phillips, 1965) or to the temporal distinctiveness of terminal items (e.g., Brown et al., 2007 ; Glenberg \& Swanson, 1986 ; Neath, 1993).

Another type of recency emerges from examination of lag-conditional response probabilities (lag-CRPs) which conditionalize recall probability on the lag $j-i$ between the serial positions $i$ and $j$ of two successive recalls. This analysis of recall contingencies has revealed two consistent behaviors (Howard \& Kahana, 1999 ; Kahana, 1996 ; Kahana, Howard, Zaromb, \& Wingfield, 2002): First, a recalled item tends to be followed by items from nearby in the list, such that the lag-CRP function declines with increasing lag; this is referred to as lag-recency. To date, all reported lag-CRP functions have exhibited this regularity, with smaller transitions always more likely than transitions involving greater lags. Second, the lag-CRP functions are asymmetric, with a transition of a given positive lag being more likely than a transition with a negative lag of the same magnitude. The proximity and asymmetry of transitions in the lag-CRP function are obtained even when accounting for the constraint that there are fewer opportunities to make transitions of greater distance (Howard \& Kahana, 1999).

The proximity and asymmetry of transitions during free recall constitute two empirical regularities that have guided recent theorizing (e.g., Davelaar, Goshen-Gottstein, Ashkenazi, Haarmann, \& Usher, 2005 ; Howard \& Kahana, 1999, 2002a). In particular, the nature of the lag-CRP functions in free recall, along with recency over various time scales, has been central to the development of the Temporal Context Model (TCM; Howard \& Kahana, 2002a ; Howard, Fotedar, Datey, \& Hasselmo, 2005). In common with other models of episodic memory (e.g., Brown, Preece, \& Hulme, 2000 ; Dennis \& Humphreys, 2001 ; Glenberg et al., 1980), TCM assumes a critical role for the association between items and the temporal context in which they occur. At study, items are associated to a continuously changing context signal, and the context signal in turn is used to retrieve items during recall.

A fundamental assumption of TCM is that context is retrieved: At any point in time, the current context signal is assumed to be a composite of the previous state of the context and the context that was retrieved in response to the item presented or recalled (Howard \& Kahana, 2002a). This core property of TCM gives rise to two principal predictions: First, the first item recalled is expected to come from terminal list positions because the context at the beginning of recall most closely matches the items just presented. Second, all subsequent recalls are expected to involve neighboring list positions because the retrieved context overlaps most with list neighbors (Howard \& Kahana, 2002a). Although TCM is not a fully-specified model of free recall (Howard \& Kahana, 2002a), it is ideally poised to describe the evolution of temporal context in models that have thus far only assumed its presence without describing how it evolves (e.g., Farrell, 2006 ; Lewandowsky \& Farrell, 2008 , in press).

In this article, we re-examine both the data that have propelled development of TCM and the theory's predictions. To anticipate our conclusions, we show that existing data analyses have been limited and TCM's assumptions inconsistent, thus potentially fostering misconceptions about (a) the nature of free recall, and (b) TCM's ability to account for 
people's behavior. Specifically, we first re-analyze 14 free-recall data sets and show that the lag-CRP functions are in fact frequently non-monotonic if lags outside the narrow window considered to date are included. We then show that TCM, as implemented to date, is unable to handle the widespread non-monotonicity of lag-CRP's. We next note that all existing applications of TCM have used two mutually inconsistent context signals to produce the two phenomena of interest, namely first-recall probabilities (FRP's; the serial position of the first recalled item) and lag-CRP functions. We next present a modified version of TCM that resolves this inconsistency, and show that the behavior of this modified version qualitatively departs from that of the extant model. Although this modified model can produce non-monotonicity in lag-CRP's, it still cannot convincingly handle the existing data. We conclude that TCM must await additional development before it can advance our understanding of free recall processes.

\section{Re-analysis of lag recency effects in free recall}

To date, published lag-CRP functions have been computed over a limited range of lags, typically ranging from -5 to +5 (Howard et al., 2005 ; Howard \& Kahana, 2002a, 2002b ; Howard, Kahana, \& Wingfield, 2006 ; Kahana, 1996 ; Kahana \& Howard, 2005 ; Kahana et al., 2002 ; Klein, Addis, \& Kahana, 2005 ; Sirotin, Kimball, \& Kahana, 2005 ; Zaromb et al., 2006 ) or, in a few cases, ranging from -6 to +6 or more (Howard \& Kahana, 1999 ; Howard, Youker, \& Venkatadass, in press ; Howard, Venkatadass, Norman, \& Kahana, in press). Kahana (1996) justified the focus on this restricted range by citing the smaller number of available observations at longer lags. Nonetheless, the focus on lags $\leq 6$ has excluded a large proportion of data from consideration (up to $30 \%$ of all non-adjacent transitions; see Table 1). As these excluded data may provide important additional information about transitions in free recall, we re-analyzed 5 experiments with 14 different conditions that have provided most extant lag-CRP functions: Experiments 1 and 2 of Howard et Kahana (1999); Murdock et Okada (1970); the 6 conditions of Murdock (1962); and lists from Howard, Venkatadass, et al. (in press) not containing repetitions. All possible transition lags were included in the analyses. To underscore the relevance of this re-analysis, Table 1 shows the number of transitions in those 14 data sets broken down by lag (considering only the first two output positions for reasons noted later). The table shows the number of transitions at lag 1; at lags 2-5; and at "extreme" lags greater than 5 that have hitherto been excluded. The table also summarizes the prevalence of extreme lags: $P_{\text {ext }}$ refers to the proportion of extreme lags out of all non-adjacent transitions, and $R_{\text {ext }}$ refers to the ratio of extreme lags and those at intermediate transitions (i.e., 2 - 5). The proportions of hitherto-excluded data are sufficiently large $\left(P_{\text {ext }}\right.$ is .30 on average for positive lags and .29 for negative lags) to warrant examination.

All our analyses (and data-fitting below) proceeded as follows. First, because Kahana (1996) noted that lag-CRP functions are steepest for the first two output positions, we considered only these responses for every participant-trial; this also ensures consistency between the simulations we present later and the fits presented in Howard et Kahana (2002a). ${ }^{1}$

Second, because we were interested in the transition from one correct report to the

\footnotetext{
${ }^{1}$ None of the conclusions offered in this article change materially if the analyses or model-fitting include all output positions.
} 
next, trials that included a repetition, an omission, or an extra-list intrusion among the first two responses were excluded. Third, all lag-CRP functions were computed using chancecorrected proportions; that is, during aggregation across trials and participants, we took into account the set of all possible transitions for each observed actual transition. All lagCRP plots shown in this article are based on these chance-corrected transition probabilities. 2

An illustrative result is shown in Figure 1 (Howard \& Kahana, 1999, delayed recall; data are represented by crosses). Although lag-CRP's decline monotonically within the window \pm 5 , the figure also reveals strong evidence for non-monotonicity at greater lags in some of the panels. Statistical confirmation of non-monotonicity was obtained by fitting four candidate descriptive functions to all observed lag-CRP's. The four candidates consisted of two monotonic functions (an exponential and a power function) and two non-monotonic functions (a quadratic and a composite of complementary exponentials). Formally, as a function of absolute lag $l$, the exponential function was:

$$
f(l)=\exp (-a l)
$$

where $a$ is a free parameter. The power function was given by:

$$
f(l)=l^{-a} .
$$

For the quadratic,

$$
f(l)=a+b l+c l^{2},
$$

with free parameters $a, b$, and $c$. Finally, the function involving complementary exponentials incorporated one increasing and one decreasing exponential function across lags, the latter to capture any upturns in transition probabilities at extreme lags, and was defined as:

$$
f(l)=c \exp (-a l)+(1-c) \exp [-b(L-l)],
$$

where $L$ is the list length, and $a, b$, and $c$ are free parameters. Note that the argument for the second exponential $(L-l)$ runs "backwards," thus reversing the exponential decrease with lag into an exponential increase.

Parameters were estimated by fitting each candidate function to each of the empirical lag-CRP functions for the 14 data sets listed in Table 1. Positive and negative lags were fit separately and independently. Because the large discontinuities between lags 1 and the remainder of the lag-CRP's (which are evident in Figure 1 and were present in all data sets) might have unduly favored the more complex non-monotonic functions even if the data were monotonic, the fits only considered lags greater than $1 .^{3}$ The solid lines with circles as plotting symbols in Figure 1 represent the best-fitting descriptive model; a circled "U" in a panel indicates that the best descriptive function was non-monotonic (i.e., either quadratic or the complementary-exponentials function). The figure shows that in 5 out of 8 cases, the best-fitting function was non-monotonic.

\footnotetext{
${ }^{2}$ Further details about how transitions were corrected for chance can be found in the supplementary material for this article at the web address provided at the end of this article.

${ }^{3}$ None of the conclusions are substantially altered if lags \pm 1 are included in the fitting.
} 
To provide a concise summary of the 14 data sets, the models were compared for each data set using Akaike weights (Burnham \& Anderson, 2002 ; Wagenmakers \& Farrell, 2004) obtained from the AIC statistic (Akaike, 1974). The AIC statistic allows comparison between models of differing complexity because it trades off goodness-of-fit and the number of parameters that need to be estimated to achieve that fit. Akaike weights are derived from AIC values but have the additional advantage that they can be interpreted as conditional probabilities; that is, the probability that a given model is best given the data and the set of candidates. Table 2 compares the Akaike weights for the candidate functions for all 14 data sets for negative and positive lags separately. The best model for each data set is identified by a bold-faced Akaike weight. Figures for individual experiments are available in the web-based supplementary material.

Table 2 suggests a clear conclusion: In many cases the lag-CRP functions are clearly not monotonic, and across all data sets, the average Akaike weight for the complementaryexponentials function (.58 for backward and .52 for forward transitions) favors a nonmonotonic characterization of the data. To illustrate this overall non-monotonicity, Figure 2 displays the average lag-CRP function for the 14 re-analyzed data sets. The plotted points within the \pm 5 window were formed by averaging the observed transitions at those lags across data sets; the points outside the window were formed by outside-justified alignment. Specifically, the greatest lag shown in the figure $( \pm 9)$ represents the largest transition possible in the data set with the shortest list length (condition 10-2 of Murdock, 1962); all other data sets were aligned so that their most extreme transition contributed to the average of lag \pm 9 and so on down to \pm 6 . A clear rise in transitions for extreme lags is evident; in the forward direction, this rise is as large as the drop across intermediate (2-5) lags.

\section{Summary}

A re-analysis of free-recall data from 644 participants comprising some 17,500 responses failed to yield consistent evidence for a monotonic decrease in transition probability with increasing lag. We confirmed the observation that immediate transitions $( \pm 1)$ were uniformly more likely than more remote transitions (Kahana, 1996 ; Laming, 1999). We also observed the expected monotonic decline across all lags in some of our analyses (see Table 2). However, on balance, when all data are considered together (see Figure 2), there was a clear non-monotonicity of the lag-CRP functions with an upturn in transition probabilities for lags greater than 5 or 6 . These "extreme" lags comprise a significant proportion of all responses (see Table 1), suggesting that their dismissal in summarizing people's free-recall performance is inadvisable. It follows that the monotonic decline of transition probabilities with lag, widely implied in previous work based on a subset of the available data, does not always adequately characterize people's free recall performance.

This non-monotonicity can best be understood as the effects of primacy and recency revealing themselves in the lag-CRP function. For example, the upturn in the forward direction indicates that regardless of the item just recalled, the next item tends to come from the last few list positions. Because our focus here is on the implications of our reanalysis for TCM, we do not seek to explain what might cause the presence or absence of non-monotonicity; identification of the relevant variables remains an obvious endeavor for future research. We next quantitatively examine TCM's account of these re-analyzed data, and show that there is no consistent set of assumptions that allows TCM to capture these 

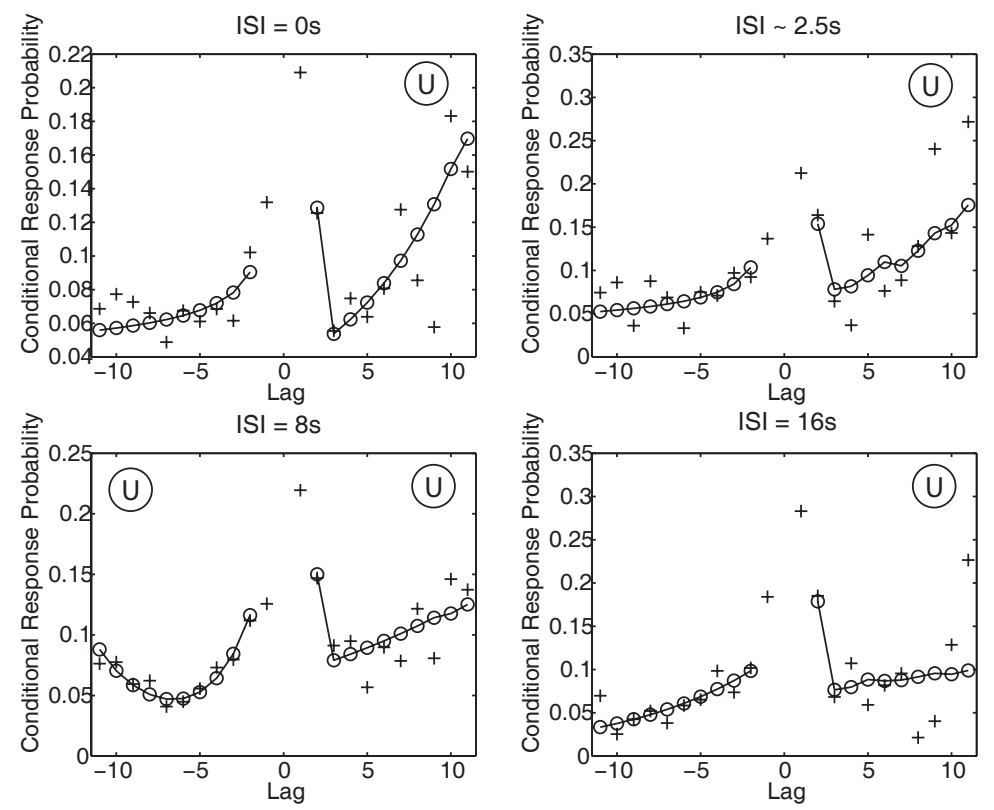

Figure 1. Lag-CRP functions for Experiment 2 of Howard \& Kahana (1999), along with predictions of the descriptive model with the highest Akaike weight for forward and backward transitions. Panels correspond to different durations of continuous distraction between items; in all cases a $16 \mathrm{~s}$ distractor task intervenes between list presentation and delayed recall. Crosses depict data, while curves show the predictions of the model for transition distances $>1$. A circled " $U$ " in the top left or top right of a panel indicates that the best-fitting curve is non-monotonic, for backward and forward transitions respectively.

results.

\section{Recency and lag-recency in TCM}

\section{Status and scope of TCM}

Our critique must begin by analyzing the status of TCM and its intended scope. This is particularly relevant because in an introductory presentation, Howard et Kahana clearly stated that "TCM is not a free recall model" (2002a, p. 293); instead, the model was presented as a description of limited aspects of free recall, namely recency (encompassed by FRP's) and associative effects (encompassed by lag-CRP's). Notwithstanding, support for TCM has been adduced by its application to those features of free-recall performance since its inception. To date, explorations of the model have addressed its predictions concerning recency over various time scales (Howard, 2004), they have demonstrated its account of the 


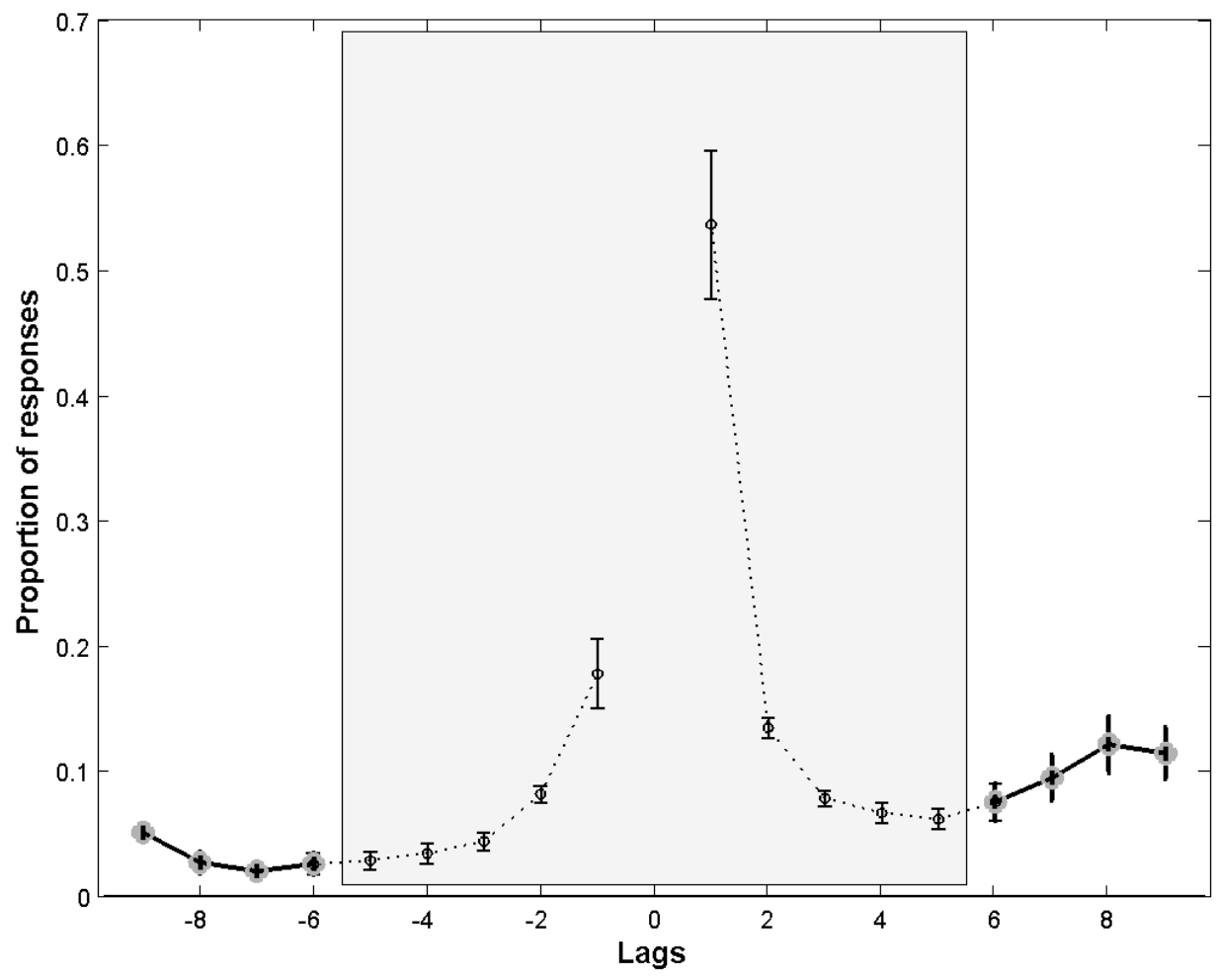

Figure 2. Average observed lag-CRP functions for all 14 data sets. "Extreme lags" outside the shaded box are outside-justified; see text for more explanation. Error bars represent standard errors across $N=14$ data sets. 
effects of ageing on recency and lag-recency (Howard et al., 2006), and they have extended its scope to spatial navigation (Howard et al., 2005). In all cases, the match between data and predictions has been taken in support of the model. We conclude that irrespective of what exactly TCM is a model of, the fact that it is supported by data must imply that it can also be challenged by those same (re-analyzed) data.

Concerning scope, Howard (2004) stated that the "TCM ... has been used to describe the recency effect and associative effects in immediate, delayed and continuous-distractor free recall experiments" (p. 234). Although this fairly unequivocally implies that FRP's and lag-CRP functions at all retention intervals are within the purview of TCM, to date all published lag-CRP applications have been limited to delayed recall. In immediate recall, the model has been shown to predict FRP's (e.g., Howard \& Kahana, 2002a) but modeling of lag-CRP functions has been deemed unsuitable because "[i]n immediate free recall, the CRP is not stable across output positions ..., making it a less-than-ideal environment for asking basic questions about contiguity effects" (Howard \& Kahana, 2002a, p. 283). We conclude that TCM was designed to describe recency and contiguity effects at all time scales (cf. abstract of Howard \& Kahana, 2002a), although lag-CRP functions for immediate recall have thus far been exempted from consideration. This exemption has been justified by properties of the data rather than on an in-principle inapplicability of TCM. As a consequence, TCM's account of lag-CRP's for immediate recall is undocumented. One purpose of this paper is to present a thorough exploration of the behavior of TCM in immediate recall.

\section{Implementations of TCM}

The core assumption of TCM, and a source of its attractiveness, is that human memory relies on a continuously evolving temporal context; in contrast to the family of models in which temporal context drifts randomly (Davelaar et al., 2005 ; Estes, 1955 ; Murdock, 1997), TCM assumes that the evolution of context is driven by the encoding and retrieval of items. In consequence, recent episodes are themselves used to create a context for the encoding of new episodes. This elegant mechanism comes at the cost of some representational and descriptive complexity which requires careful differentiation between different components of context. To facilitate understanding of our analysis of TCM we now introduce some terminology that is used in the remainder of this article.

When an item is presented for learning at time $i$, it is assumed to be bi-directionally associated with the current temporal context, which in turn is the weighted sum of two components. The first component is the context that prevailed at time $i-1$, called carryover context from here on. The second component is the context that was retrieved by the item presented at time $i-1$. This retrieved context, $\mathbf{t}_{I N}$, in turn involves a combination of pre-experimental context(s) and contexts associated with the item during the experiment. At test, context is used to cue retrieval; following recall of an item, the temporal context once again evolves in the same manner as for stimulus presentation.

A crucial implication of this mechanism is that recall at time $t$ is cued by the weighted combination of the carry-over context (from time $t-1$ ) and the retrieved context elicited by the just-recalled item; the relative contribution of those two cue components turns out to be critical in determining the model's predictions.

Both recency and lag-recency follow from these core assumptions about context in 
TCM. The recency effect follows from the overlap between the context at the end of list presentation and the context used to initiate recall; if it is assumed that the list-final context will carry over into recall, there will naturally be a close match between the recall context and the temporal context associated with items towards the end of the list (cf. non-reinstatable context in Brown et al., 2000). In consequence, the first item recalled will almost invariably be from the last few list positions, thus giving rise to steep recency in the FRP function.

Concerning lag-recency, the retrieved context component $\mathbf{t}_{I N}$ allows the model to predict a decline in lag-CRP's with increasing lags: When item $i$ is recalled and used to evolve the temporal context, it will retrieve a context that will partially match the contexts associated with items $i-1$ and $i+1$, with increasingly poorer matches for increasing lag $j$ between item $i$ and item $i \pm j$. As currently presented, TCM necessarily predicts that the conditional transition probabilities will fall off monotonically with increasing lag (see, e.g., Equation 31 of Howard, 2004).

Although the evolution of context represents the conceptual core of the model (Howard \& Kahana, 2002a, p. 293, summarize TCM as "a model that prescribes a set of rules for how a distributed episodic representation should change from moment to moment"), in actual fact contextual evolution has not been instantiated for any of the predictions published to date. For that reason, the forthcoming discussion differentiates between the published implementation of TCM (henceforth, $\mathrm{TCM}_{p u b}$ ) and a novel implementation, created for the purposes of this article, which incorporates the evolving context $\left(\mathrm{TCM}_{\text {evo }}\right)$ that has hitherto only been described verbally. ${ }^{4}$

We first fit $\mathrm{TCM}_{p u b}$ to the re-analyzed data and, in the process, point out how the instantiation to date has circumvented the evolution of context that forms the conceptual core of the theory. We then present $\mathrm{TCM}_{\text {evo }}$ and show how its predictions differ from that of the published version, before applying $\mathrm{TCM}_{\text {evo }}$ to the re-analyzed data.

\section{Standard implementation: $T C M_{p u b}$}

$\mathrm{TCM}_{p u b}$ was fit to the re-analyzed data using maximum likelihood estimation. Following Howard et Kahana (2002a), and paralleling our re-analysis, we fit only the first two output positions. The first output position determines the FRP's, and the transition between the two output positions determines the lag-CRP. Maximum likelihood parameter estimates were obtained for each participant separately using the Simplex algorithm (Nelder \& Mead, 1965). Likelihood statistics for all data sets (summed across participants in each case) are given in Table 3. To provide an indication of the model's performance, the final column in the table provides the critical values of $\chi^{2}$ (the deviance, $-2 \ln L$, approximates a $\chi^{2}$ distribution). The mean parameter estimates associated with the fits (averaged across participants) are given in Table 4.

Across all data sets, the match between $\mathrm{TCM}_{p u b}$ and the empirical FRP's varied. Representative fits are shown in Figure 3. The left panel shows the fit to the $I S I=16$ condition in Experiment 2 of Howard et Kahana (1999); for these and other data (e.g., Howard \& Kahana, 1999 ; Howard, Venkatadass, et al., in press), the model gave a good account of the first recall probabilities. By contrast, for the data of (Murdock \& Okada,

\footnotetext{
${ }^{4}$ To conserve space, a formal description of both models and their fit to empirical data is given in the web-based supplementary material, along with figures for all model fits.
} 

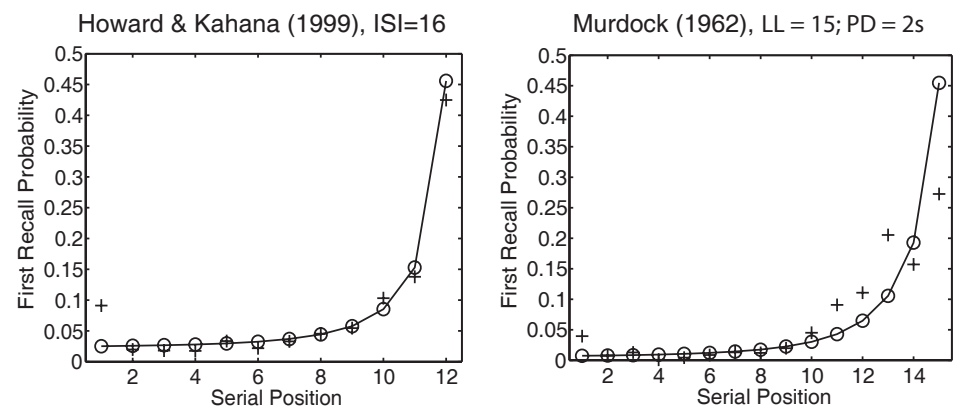

Figure 3. Illustrative fits of $\mathrm{TCM}_{p} u b$ to FRP's. Left panel: Fit to the data from the $I S I=16$ condition in Experiment 2 of Howard \& Kahana (1999); right panel: Fit to the data from the list length $=15$, presentation duration $=2 \mathrm{~s}$ condition of Murdock (1962).

1970) and (Murdock, 1962), the model over-predicted the recency in the FRP's; this misfit is illustrated in the right panel of Figure 3.

Concerning lag-CRP's, in most cases the model's predictions did not accord with the empirical results. Figure 4 shows representative predictions of $\mathrm{TCM}_{p u b}$ for the data shown earlier in Figure 1 (Recall that all panels involve delayed recall but with different interstimulus intervals involving a continuous distractor task). Across all data sets, $\mathrm{TCM}_{p u b}$ often under-predicted the occurrence of +1 transitions; in Figure 4 this is illustrated in the bottom-right panel for the $16 \mathrm{~s}$ ISI condition. The model also consistently failed to capture the non-monotonicity in the data, as is evident from all panels of Figure 4. Note, however, that the model's predictions are roughly in accord with its previously published predictions, except that here the predicted lag-CRP functions span a wider range of lags and are more likely to under-estimate the occurrence of +1 transitions.

The deviation between predictions and data in the FRP's and the lag-CRP's can be attributed to the heavy tails and frequent non-monotonicity in the empirical lag-CRP's. In order to handle the large number of extreme transitions, $\mathrm{TCM}_{p u b}$ produced relatively flat lag-CRP functions, at the expense of under-predicting the \pm 1 transitions. The only 

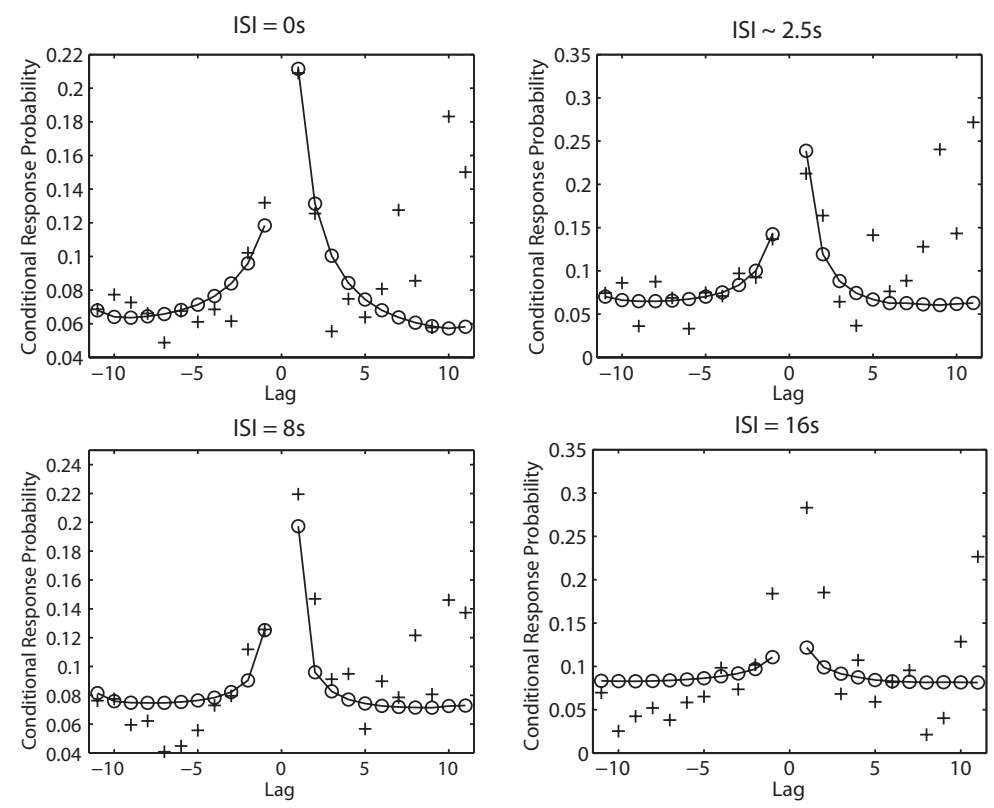

Figure 4. Lag-CRP functions predicted by $\mathrm{TCM}_{p u b}$ (connected circles; crosses are data) for the no delay (top-left), 2.5s delay (top right), 8s delay (bottom left) and $16 \mathrm{~s}$ delay (bottom right) conditions in Experiment 2 of Howard and Kahana (1999).

way in which the model can flatten the predicted lag-CRP functions is by decorrelating the successive list contexts by increasing the contribution of retrieved context. Given the trade-off between carry-over and retrieved context, one consequence is the excessive recency in the predicted FRP's.

In summary, as currently instantiated, $\mathrm{TCM}_{p u b}$ is qualitatively and quantitatively unable to accommodate the data. In particular, the model fails to capture the clear nonmonotonicity in the lag-CRP's. This problem has remained undiscovered to date because analysis and modeling have been restricted to a relatively narrow window of lags.

In addition to noting the model's mispredictions, the manner in which those predictions are obtained also deserves clarification. In all applications to date, Howard, Kahana, and colleagues make a simplifying assumption that, though subtle, has significant implications. Specifically, $\mathrm{TCM}_{p u b}$ assumes that at the outset of recall, the context cue is identical to the context prevailing at the end of list presentation (Howard \& Kahana, 2002a). In consequence, the first item recalled will almost invariably be from the last few list positions, thus giving rise to steep recency in the FRP function. If post-list distractors are present, context is updated after list-presentation and the pre-recall context is no longer 
identical to the context for the last list item, thus generating the reduction in recency with distractor-filled delays.

By contrast, when accounting for the lag-CRP functions in $\mathrm{TCM}_{p u b}$, Howard and colleagues have consistently assumed that the carry-over context, which lingers from the previous recall, is orthogonal to all contextual states from the list (Howard \& Kahana, 2002a ; Howard, 2004); this is equivalent to assuming that an infinitely long time has intervened between the list and the recall attempt to be modeled. In consequence, the model necessarily predicts monotonically decreasing lag-CRP functions because the retrieved context, elicited by the just-recalled item, provides the sole basis on which to discriminate between potential recall candidates; the compound context cue thus preferentially elicits list items proximate to the immediately preceding recall.

It follows that $\mathrm{TCM}_{\text {pub }}$ 's predictions for FRP's and lag-CRP's, respectively, are obtained under two very different circumstances. When predicting FRP's, the context prevailing at the end of study (or its updated derivative) is used as a cue; when predicting lag-CRP's, the carry-over context is not actually carried over between recalls, but is instead assumed to be orthogonal to the temporal contexts associated with all list items. The use of two distinct assumptions about the relevant context cue creates a gap between the stated deep properties of the model and its implementation. On the one hand, an appealing core property of the model is the notion of a context that gradually evolves by combining the carry-over context with the retrieved context. On the other hand, in all applications to date, the model's predictions were not derived in this fashion when accounting for the lag-CRP function. Irrespective of the quality of the fits of $\mathrm{TCM}_{p u b}$, this disconnection between the verbal description of the model and its actual implementation undermines the use of the model as a conceptual tool.

To date, there has been no published simulation that shows TCM's predictions when it is permitted to let context evolve from the state at the end of list presentation to its final (possibly orthogonal) state by continuously updating it with the contexts retrieved by the recalled items. It is possible that TCM will behave quite differently when it is computationally implemented as it is described verbally; accordingly, we next present a modified version of TCM in which context evolves gradually across list presentation and recall.

Recency and lag-recency with evolving context: $T C M_{\text {evo }}$

Demonstrations of TCM with continuously evolving context. What happens when the appropriately evolved context is used in place of an orthogonal carry-over context to predict lag-CRP functions? Assuming a continuously evolving context throughout list presentation and recall required some re-expression of the model because extant solutions only apply when there are no repetitions of items. These solutions are insufficient here because once an item has been recalled from a list, it has effectively been repeated. Those modifications are embodied in a new instantiation introduced here, $\mathrm{TCM}_{\text {evo }}$, which is detailed in the web-based supplementary material. Briefly, unlike the published version, $\mathrm{TCM}_{\text {evo }}$ uses the context retrieved by the first-recalled item in conjunction with the proper carry-over context to produce the compound cue for the second retrieval.

An initial examination of the model revealed a striking non-monotonicity of the forward lag-CRP functions in $\mathrm{TCM}_{\text {evo }}$. Irrespective of whether recall was immediate or delayed 
or involved a continuous distractor task, lags greater than 5 attracted nearly as manyor indeed more - transitions than lags +1 . This striking upturn resulted from the use of carry-over context in this model, in contrast to $\mathrm{TCM}_{p u b}$. In $\mathrm{TCM}_{p u b}$, the retrieved context elicited by the first-recalled item is combined with an orthogonal (and hence noninformative) carry-over context - in consequence, this combined context is dominated by that associated with (and retrieved by) the previously-recalled item, which gives rise to lag-recency for obvious reasons. In $\mathrm{TCM}_{\text {evo }}$, by contrast, the retrieved context is combined with a temporally-informative carry-over context that - early in recall - necessarily overlaps with the end-of-list context. Hence, cuing with the combined context tends to elicit either a neighboring item (lag-recency, driven by the retrieved component) or an item towards the end of the list (recency, driven by the carry-over component), thus necessarily giving rise to extreme non-monotonicity and U-shape of the predicted lag-CRP functions.

This is a noticeable characteristic of $\mathrm{TCM}_{\text {evo }}$ even for delayed recall: Our initial examinations revealed that even after updating the temporal context with an additional 7 items before initiating retrieval (as we assumed for delayed recall, approximating the value in Howard \& Kahana, 2002a), the model still shows a strong tendency to make extreme transitions. Both qualitatively and quantitatively, this behavior of $\mathrm{TCM}_{\text {evo }}$ deviates at all time scales from the principle of contiguity that has been proposed by Kahana (1996) and Howard et Kahana (1999).

Applying TCM with continuously evolving context to data. Although the implementation of a continuously-evolving context yields predictions that differ considerably from existing published applications of TCM, the non-monotonicities observed in our earlier reanalysis of the data are at least in tentative qualitative agreement with $\mathrm{TCM}_{\text {evo }}$ 's predictions as just described. We therefore fit $\mathrm{TCM}_{\text {evo }}$ to the re-analyzed data.

The maximum likelihood statistics for the fits of $\mathrm{TCM}_{\text {evo }}$ are shown in the right column of Table 3, and the corresponding mean parameters are given in Table 4. Representative predictions of $\mathrm{TCM}_{e v o}$ are shown in Figures 5 and 6 . Although $\mathrm{TCM}_{\text {evo }}$ was able to produce the non-monotonicity in the lag-CRP functions (in some cases excessively so; see bottom right panel of Figure 5), this often occurred at the cost of under-predicting the standard lag-recency effect (that is, transitions of small lag, particularly immediate \pm 1 transitions; see bottom right panel of Figure 5 and right-hand panel of Figure 6).

The simulations showed that when context is assumed to evolve continuously throughout list presentation and recall, $\mathrm{TCM}_{\text {evo }}$ produces behavior that is somewhat more in accord with the (re-analyzed) pattern in the data. $\mathrm{TCM}_{\text {evo }}$ correctly (albeit often excessively) predicted non-monotonicity in the lag-CRP functions, particularly in the forward direction. Accordingly, the quantitative fit of $\mathrm{TCM}_{\text {evo }}$ was also consistently better, in every instance, than the fit of $\mathrm{TCM}_{p u b}$; see Table $3 .^{5}$ On the basis of the disparity between the implementation of $\mathrm{TCM}_{p u b}$ and the verbal description of TCM, and the statistical improvement in the model when its implementation is brought in to line with that verbal description by assuming a continuously evolving context, we reject $\mathrm{TCM}_{p u b}$ and consider it no further. Instead, we focus on $\mathrm{TCM}_{\text {evo }}$ in the remaining analyses and discussion.

\footnotetext{
${ }^{5}$ The same pattern of results is observed when all legitimate responses, not just those from the first two output positions, are used to fit the model. An additional set of simulations (which we do not report to conserve space) fitting all output positions leads to the same conclusions.
} 

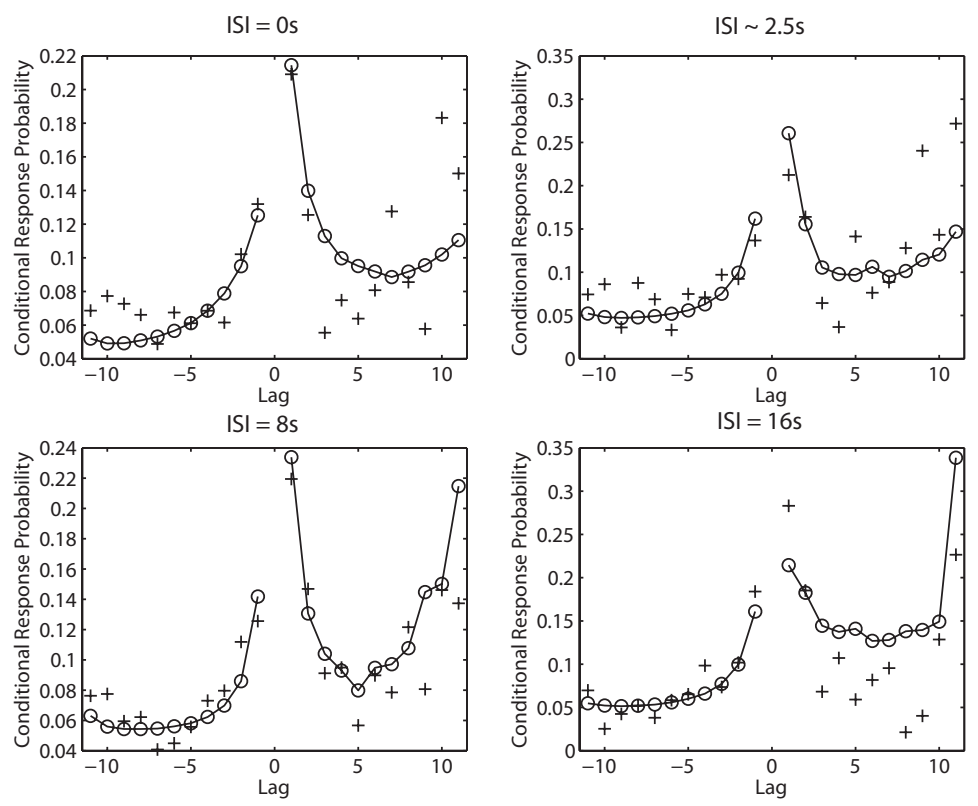

Figure 5. Lag-CRP functions predicted by $\mathrm{TCM}_{\text {evo }}$ (connected circles; crosses are data) for the $\mathrm{ISI}=0$ (top-left), $2.5 \mathrm{~s}$ (top right), $8 \mathrm{~s}$ (bottom left) and $16 \mathrm{~s}$ (bottom right) conditions in Experiment 2 of Howard and Kahana (1999).

Recency in $T C M_{\text {evo }}$. We noted at the outset that one reason for the exclusion of lags in excess of \pm 5 from all extant analyses has been the presumed scarcity of more extreme transitions. A scarcity of data has presumably also dictated another simplification of analyses to date; viz. the aggregation of lag-CRP's irrespective of the serial position of the preceding item. This aggregation is unfortunate: If the lag-recency effect truly relates to the conditional dependence between successive recalls, the effect should be present for individual serial positions of the just-recalled item. Some evidence that this is the case was presented by Laming (1999), who showed that recall of items from the first position, and positions 14-18, in the experiment of Murdock et Okada (1970) tended to be followed by recall of their respective successor. Although lag-CRP data broken down by serial position may be noisier than the aggregated data, we present a serial-position based analysis for a data set composed of a very large number of responses. The left column of Figure 7 presents lag-CRP functions for individual serial positions observed in immediate (top) and delayed (bottom) recall. The immediate recall data are from Howard, Venkatadass, et al.'s (in press) large-scale experiment involving 293 participants, and thus give reasonably smooth lag-CRP functions (the aggregate data are shown in Figure 6). It is clear from this panel 

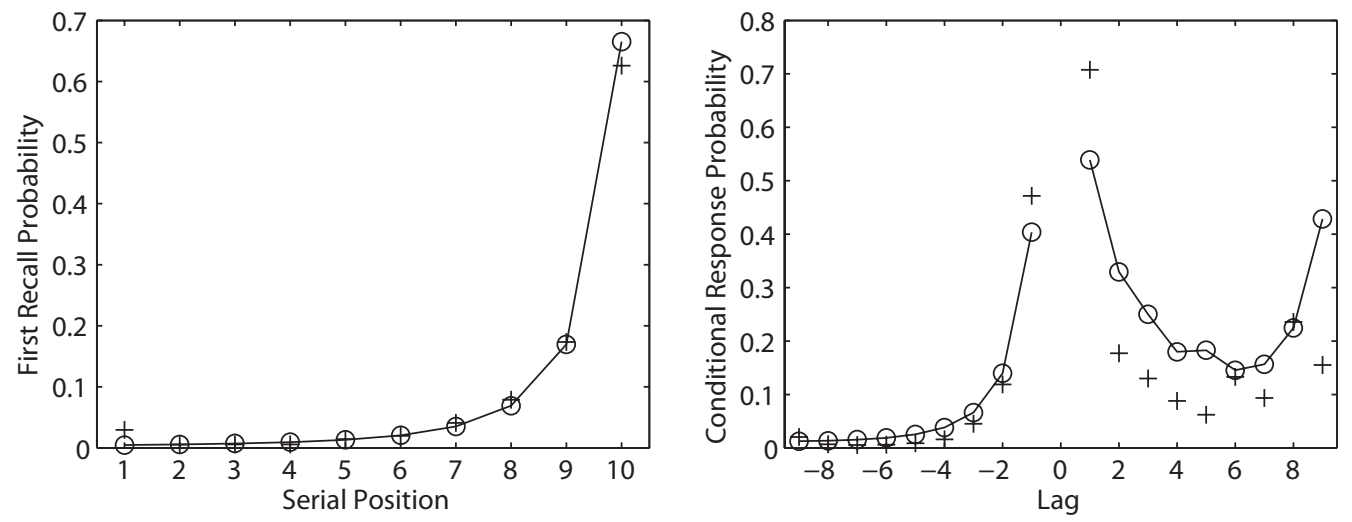

Figure 6. FRP (left panel) and lag-CRP (right panel) functions predicted by $\mathrm{TCM}_{\text {evo }}$ (connected circles; crosses are data) for the immediate free recall data of Howard, Venkatadass, et al. (in press).

that the lag-CRP functions for individual serial positions each possess similar characteristics to the aggregate data, showing increased frequencies of local (i.e., \pm 1 ) transitions and a general increase in the frequencies of extreme transitions compared to intermediate lags. The delayed recall data in the bottom left panel are from Experiment 1 of Howard et Kahana (1999); although the smaller number of responses yields noisier lag-CRP functions, they are consistent with the aggregate data (not shown here).

This consistency in the data contrasts with the lag-CRP's obtained from $\mathrm{TCM}_{\text {evo }}$. The top right panel of Figure 7 shows that, for forward transitions in immediate recall, $\mathrm{TCM}_{\text {evo }}$ does not predict any lag recency effect for individual serial positions. Instead, the predictions are characterized solely by recency: Regardless of which item has just been recalled, one of the last few list items is likely to be recalled next. The model's predictions are therefore in opposition to the regularity in the data: Instead of predicting a large decrease and a small upturn with increasing forward transitions, the model predicts a large monotonic increase. This is not the case for delayed recall, where the model correctly predicts a conventional monotonically-decreasing lag-recency effect at each serial position, along with a slight non-monotonicity for extreme transitions.

It should be noted that these predictions of $\mathrm{TCM}_{\text {evo }}$ were derived under the best- 
Immediate
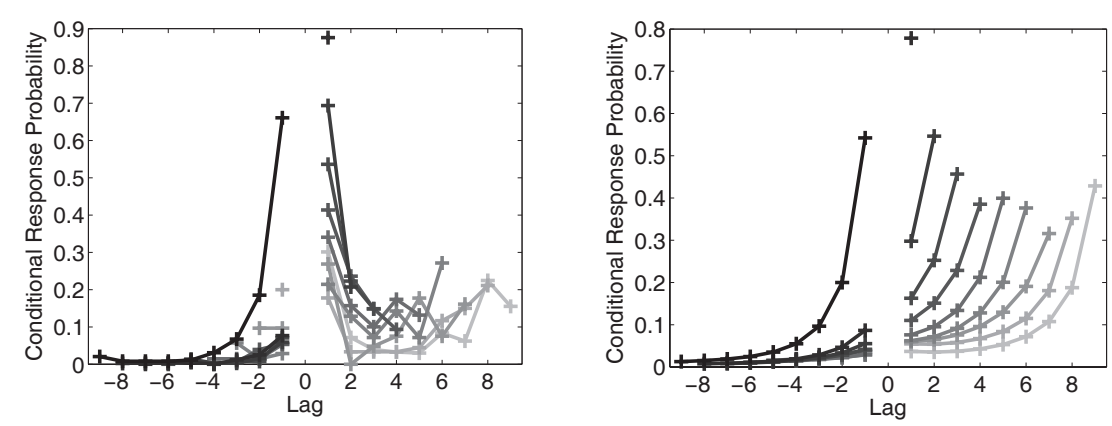

Delayed
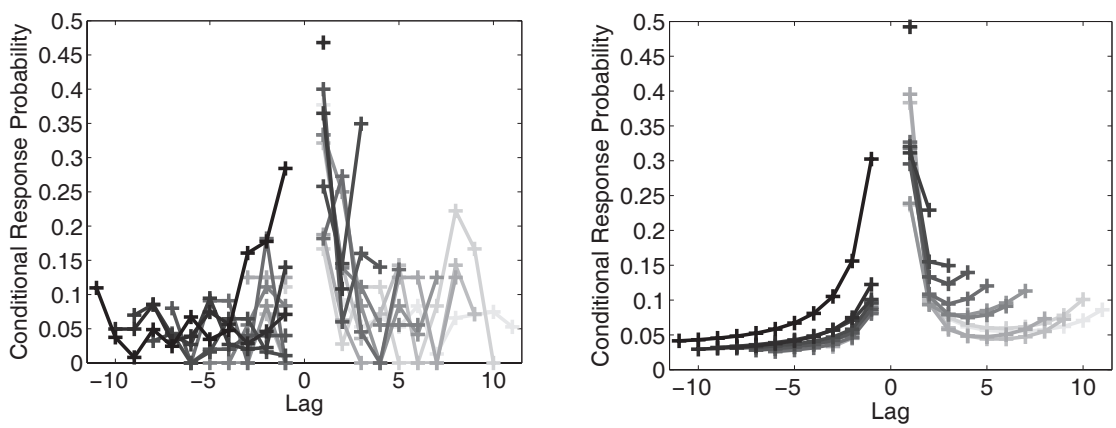

Figure 7. Lag-CRP functions for immediate recall (top panels) and delayed recall (bottom panels), broken down by the serial position of the previously recalled item. The darkest line represents the case in which the last item was recalled first (and is only present for negative lags because no forward transitions are possible after recall of the final item), and successively lighter lines represent successive serial positions back in the list of the first-recalled item (the single data point represents the case in which the penultimate list item is recalled first, for which the only possible forward transition is +1). Data are shown on the left, and $\mathrm{TCM}_{\text {evo }}$ 's predictions are shown on the right. Immediate recall data are from Howard, Venkatadass, et al. (in press); delayed recall data are from Experiment 1 of Howard \& Kahana (1999). 
fitting parameters from the immediately preceding simulations. One objection might be that if the model were fit to the individual curves in Figure 7, rather than the aggregate curves, it would display behavior more in line with the empirical results. As detailed in the supplementary materials, $\mathrm{TCM}_{\text {evo }}$ was fit by calculating, for each response, the likelihood of that response given its serial position, its output position, and the serial position of any prior recalls on that trial. It follows that these are the best possible fits that $\mathrm{TCM}_{\text {evo }}$ could give to the data as they are analyzed in Figure 7, and that this behavior is obtained under realistic parameter values.

How is $\mathrm{TCM}_{\text {evo }}$ able to qualitatively produce a conventional lag-recency effect across small lags for the aggregate analysis of immediate recall (see Figure 6), whereas the direction of this effect is mostly reversed for all constituent serial positions? The answer is that the predicted conventional lag-recency in Figure 6 is an artifact of aggregating across serial positions. There are two factors that contribute to this artifact. First, the top right panel of Figure 7 shows that the predicted probability of the maximum possible transition differs across serial positions, and that the extent of recency (as measured by the conditional recall of the last list item) roughly traces out the shape of the aggregate lag-CRP in Figure 6 . That is, the most frequent transition at each serial position is from that position to the end of the list; because the distance of that transition decreases with serial position, aggregating across serial positions contributes to an artifactual monotonic decrease of the lag-CRP function. The second contribution to this artifact results from the widely different probabilities with which items from different positions are recalled first. The predicted FRP's in Figure 6 show that most of the time, the first-recalled item will be from terminal list positions; in consequence, when lag-CRP's are aggregated across serial positions, the darker lines in Figure 7 (i.e., later positions) are weighted far more heavily than the lighter lines (early positions), which in turn engenders a monotonic decline across lags upon aggregation.

The preceding analysis of lag-CRP's by serial position is informative in three ways. First, it underscores the general importance of analyzing transition probabilities at a fine level of detail for both data and predictions. For example, the apparent ability of models to account for changes in lag-recency across output position (e.g., Davelaar et al., 2005) might simply reflect decreasing susceptibility to aggregation artifacts with increasing delay. Second, the analysis shows that, for immediate recall, the behavior of $\mathrm{TCM}_{e v o}$ is characterized solely by recency -irrespective of which item has been recalled first, the next item recalled tends to be from terminal list positions. At the level of each serial position, no set of increasing lags, however constrained or selected, produces decreasing predicted transition probabilities. We have carried out similar analyses for the remaining experiments modeled here, and in all instances of immediate recall the same behavior is produced. We conclude that the decline in transition frequency across small lags in the aggregate lag-CRP's predicted by $\mathrm{TCM}_{\text {evo }}$ arises from averaging and does not reflect the the deep property of the model. Note, though, that for delayed recall $\mathrm{TCM}_{\text {evo }}$ does qualitatively produce lagrecency in the lag-CRP functions for individual serial positions. Third, the behavior of $\mathrm{TCM}_{\text {evo }}$ identified by this analysis applies to all forward transitions, including those with lags 5 or less. It follows that this problem cannot be circumvented by questioning the generality of the non-monotonicity identified in our re-analysis.

That said, we reiterate that TCM has not previously been applied to lag-CRP functions in immediate recall. One might therefore claim that our analysis is not very informa- 
tive because it relates to a phenomenon outside the model's scope. Our response is twofold: First, existing justifications for the exclusion of immediate lag-CRP's have focused on the change of empirical CRP's with output position, and hence the data, rather than on a fundamental problem with the model. It follows that our analysis is informative, even if only proactively, because it presents a strong constraint that further model development must accommodate; continued dismissal of a reliable empirical phenomenon merely because it challenges one's model appears unwise and ultimately unsustainable. Second, given the explicit admonitions that TCM is not a theory of free recall (e.g., Howard \& Kahana, 2002a), one must be concerned by further reductions in its explanatory scope. As it stands, TCM has been identified as "... a model ... to describe the recency effect and associative effects in immediate, delayed and continuous-distractor free recall experiments" (Howard, 2004, p. 234); in light of the existence of other, widely encompassing theories of free recall (e.g., Brown et al., 2007 ; Davelaar et al., 2005) it appears unwise and ultimately unsustainable to rule that associative effects are within the purview of TCM only if accompanied by long retention intervals. Ultimately, a theory's power is proportional to what it can explain; whether it fails to explain a phenomenon (as we just showed) or whether that phenomenon is arbitrarily put outside the theory's scope (as in previous practice involving TCM) has equal limiting impact on the theory's explanatory power.

\section{General Discussion}

Our work departed from three assumptions that appeared to be solidly entrenched in the literature: (1) During free recall, when people transition from one item to the next, they favor transitions across a small number of intervening serial positions over longer ones, a tendency captured by monotonically-decreasing lag-CRP functions. (2) Since its inception, the Temporal Context Model (TCM) has reliably and repeatedly captured this pattern of lag-CRP's, at least in delayed recall. (3) TCM simultaneously accounts for the recency observed in first-recall probabilities. This article identified all three of those assumptions to be subject to qualification or revision.

First, we re-analyzed 14 data sets that have been used to adduce support for these three assumptions, and discovered that many empirical lag-CRP functions are not monotonic, contrary to the previous consensus in the literature. Our re-analysis differed from precedents by considering all possible transitions, thus including the substantial proportion of the data that had been ignored in the past. We suggest that a proper characterization of lag-CRP's in free recall must acknowledge their frequent non-monotonicity, which in turn reflects the contribution of primacy and recency effects to these conditional analyses.

Second, we confirmed by simulation that the published version of TCM cannot accommodate the widespread non-monotonicity in the empirical lag-CRP's, thus revealing a qualitative disparity between the model's predictions and the actual shape of the data which it was designed to explain.

Third, we noted that TCM has hitherto used two distinct assumptions about context to model FRP's and CRP's, respectively. In so doing, a desirable core property of the model - viz. the gradual evolution of context-has been omitted from all published modeling. This compromises the utility of the TCM irrespective of how well it might fit the data.

In response, we implemented an evolving context, in line with the verbal description of the theory, in $\mathrm{TCM}_{\text {evo }}$. This modified version performed better than $\mathrm{TCM}_{p u b}$ without how- 
ever providing a satisfactory account of the data: $\mathrm{TCM}_{\text {evo }}$ under-predicted the frequency of +1 transitions in the CRP's and vastly exaggerated the extent of non-monotonicity in the lag-CRP functions. ${ }^{6}$ Finally, a more detailed analysis of immediate recall revealed that $\mathrm{TCM}_{\text {evo }}$ 's lag-CRP predictions at the level of individual serial positions were in exact opposition to the data: Whereas the empirical forward lag-CRP's have a strong declining component, from immediate $(+1)$ transitions to moderately large lags (e.g., +5$)$, the predicted lag-CRP's are monotonically increasing. $\mathrm{TCM}_{\text {evo }}$ thus predicts that once an item has been recalled, the next item reported is from the end of the list, irrespective of the serial position of the preceding recall.

What implications do our results have for the temporal context model and for our understanding of free recall in general? Concerning the latter question, irrespective of one's preferred theoretical stance, this article suggests that the hitherto accepted consensus about the shape of lag-CRP's must be revised or at least qualified. Theoreticians can no longer assume that transitions during free recall solely involve items in close proximity; in a significant number of cases, people are more likely to transition across a large number of serial positions than an intermediate number.

Turning to the implications for TCM, we suggest that the model cannot describe the evolution of context while simultaneously accounting for monotonic recency and lag-recency effects in free recall. As Figure 5 shows, even under the most favorable parameter values, the model produces behavior that is incommensurate with either its previously published behavior or - in many instances - the data. Note that this conclusion can be drawn without considering immediate free recall; all panels in Figure 5 involve delayed recall. Does our analysis necessarily call into question the overall architecture of TCM? Might the theory be able to accommodate the data with a few simple modifications?

First, we do not believe that our analysis necessarily calls into question all core principles of the model. The recency that is observed in experiments with continuous distractor tasks certainly favors explanations based on temporal distinctiveness or contextual overlap (e.g., Brown et al., 2007 ; Davelaar et al., 2005 ; Glenberg et al., 1980 ; Glenberg \& Swanson, 1986 ; Neath, 1993), and the lag-recency effect, particularly evident in \pm 1 transitions, suggests some local associations between items on lists (direct, or mediated by temporal context; Howard \& Kahana, 1999 ; Kahana, 1996); both of these assumptions are embodied in TCM. However, in light of our simulations, the exact instantiations of these principles in the model may need to be revisited.

Second, concerning such possible modifications, we note that the problems we have identified with TCM are revealed by the very data that have constituted its principal support. In consequence, we have limited ourselves to exploring only solutions that could

\footnotetext{
${ }^{6}$ In an attempt to increase the proximity of transitions in TCM, we devised one other version which assumed that an orthogonal carry-over context was used for all recalls, including the first output position. (This basically generalized the assumption used in $\mathrm{TCM}_{p u b}$ for lag-CRP's to all output positions.) This version of the model is not presented in detail as it performed significantly worse than the other versions considered here; in particular, the model predicted perfectly flat FRP functions, which clearly does not accord with the extensive recency in the data. Thus, irrespective of whether the disjointed assumptions about temporal context in $\mathrm{TCM}_{p u b}$ are reconciled within $\mathrm{TCM}_{e v o}$ or by generalizing the orthogonality assumption to both phenomena under consideration, no common set of assumptions about temporal context enabled TCM to simultaneously handle the two phenomena-FRP's and CRP's - that provided its foundational support.
} 
arguably be implicit in TCM's current formulation. One modification, already reported, was to develop $\mathrm{TCM}_{\text {evo }}$, which instantiated available verbal descriptions of the model. We have also explored another parameterization of $\mathrm{TCM}_{\text {evo }}$ in which the parameter that governs the balance between retrieved and carry-over context was allowed to vary between study and test. ${ }^{7}$ We do not report those additional simulations because they do not materially affect our conclusions. We conclude that theoreticians may have to look further afield to enable TCM to handle lag-CRP and FRP functions in free recall.

\section{Archived Materials}

The following materials associated with this article may be accessed through the Psychonomic Society's Norms, Stimuli, and Data archive, www.psychonomic.org/archive.

To access these file(s), search the archive for this article using the journal name (Psychonomic Bulletin \& Review), the first author's name (Farrell), and the publication year (2008).

FILE: TCMsupplement.pdf

DESCRIPTION : This single text file contains a detailed description of the two models examined here, $\mathrm{TCM}_{p u b}$ and $\mathrm{TCM}_{e v o}$, further details about data fitting procedures, and the detailed results of all re-analyses and model fits reported here.

\section{Références}

Akaike, H. (1974). A new look at the statistical model identification. IEEE Transactions on Automatic Control, 19, 716-723.

Brown, G. D. A., Neath, I., \& Chater, N. (2007). A temporal ratio model of memory. Psychological Review, 114, 539-576.

Brown, G. D. A., Preece, T., \& Hulme, C. (2000). Oscillator-based memory for serial order. Psychological Review, 10\%, 127-181.

Burnham, K. P., \& Anderson, D. R. (2002). Model selection and multimodel inference: A practical information-theoretic approach (2nd Edition). New York : Springer-Verlag.

Davelaar, E. J., Goshen-Gottstein, Y., Ashkenazi, A., Haarmann, H. J., \& Usher, M. (2005). The demise of short-term memory revisited: Empirical and computational investigations of recency effects. Psychological Review, 112, 3-42.

Dennis, S., \& Humphreys, M. S. (2001). A context noise model of episodic word recognition. Psychological Review, 108, 452-478.

Estes, W. K. (1955). Statistical theory of spontaneous recovery and regression. Psychological Review, 62, $145-154$.

Farrell, S. (2006). Mixed-list phonological similarity effects in delayed serial recall. Journal of Memory and Language, 55, 587-600.

Glanzer, M., \& Cunitz, A. R. (1966). Two storage mechanisms in free recall. Journal of Verbal Learning and Verbal Behavior, 5, 351-360.

Glenberg, A. M., Bradley, M. M., Stevenson, J. A., Kraus, T. A., Tkachuk, M. J., Gretz, A. L., et al. (1980). A two-process account of long-term serial position effects. Journal of Experimental Psychology: Human Learning and Memory, 6, 355-369.

Glenberg, A. M., \& Swanson, N. G. (1986). A temporal distinctiveness theory of recency and modality effects. Journal of Experimental Psychology: Learning, Memory, and Cognition, 12, 3-15.

\footnotetext{
${ }^{7}$ We thank Marc Howard for suggesting this modification in a review of an initial version of this manuscript.
} 


\section{EMPIRICAL AND THEORETICAL LIMITS ON LAG RECENCY IN FREE RECALL21}

Howard, M. W. (2004). Scaling behavior in the temporal context model. Journal of Mathematical Psychology, 48, 230-238.

Howard, M. W., Fotedar, M. S., Datey, A. V., \& Hasselmo, M. E. (2005). The temporal context model in spatial navigation and relational learning: Toward a common explanation of medial temporal lobe function across domains. Psychological Review, 112, 75-116. Disponible sur http://dx.doi.org/10.1037/0033-295X.112.1.75

Howard, M. W., \& Kahana, M. J. (1999). Contextual variability and serial position effects in free recall. Journal of Experimental Psychology: Learning, Memory, and Cognition, 25, 923-941.

Howard, M. W., \& Kahana, M. J. (2002a). A distributed representation of temporal context. Journal of Mathematical Psychology, 46, 269-299.

Howard, M. W., \& Kahana, M. J. (2002b). When does semantic similarity help episodic retrieval? Journal of Memory and Language, 46, 85-98.

Howard, M. W., Kahana, M. J., \& Wingfield, A. (2006). Aging and contextual binding: Modeling recency and lag-recency effects with the temporal context model. Psychonomic Bulletin 8 Review, 13, 439-445.

Howard, M. W., Venkatadass, V., Norman, K. A., \& Kahana, M. J. (in press). Associative processes in immediate recency. Memory $\mathscr{E}$ Cognition.

Howard, M. W., Youker, T. E., \& Venkatadass, V. S. (in press). The persistence of memory: Contiguity effects across hundreds of seconds. Psychonomic Bulletin $\mathscr{E}$ Review.

Kahana, M. J. (1996). Associative retrieval processes in free recall. Memory \& Cognition, 24, 103-109.

Kahana, M. J., \& Howard, M. W. (2005). Spacing and lag effects in free recall of pure lists. Psychonomic Bulletin \& Review, 12, 159-164.

Kahana, M. J., Howard, M. W., Zaromb, F., \& Wingfield, A. (2002). Age dissociates recency and lag recency effects in free recall. Journal of Experimental Psychology: Learning, Memory, and Cognition, 28, 530-540.

Klein, K. A., Addis, K. M., \& Kahana, M. J. (2005). A comparative analysis of serial and free recall. Memory \& Cognition, 33, 833-839.

Laming, D. (1999). Testing the idea of distinct storage mechanisms in memory. International Journal of Psychology, 34, 419-426.

Lewandowsky, S., \& Farrell, S. (2008). Phonological similarity in serial recall: Constraints on theories of memory. Journal of Memory and Language, 58, 429-448.

Lewandowsky, S., \& Farrell, S. (in press). Short-term memory: New data and a model. In B. Ross (Ed.), The psychology of learning and motivation.

Murdock, B. B. (1962). Direction of recall in short-term memory. Journal of Verbal Learning and Verbal Behavior, 1, 119-124.

Murdock, B. B. (1974). Human memory: Theory and data. Potomac, MD : Lawrence Erlbaum Associates.

Murdock, B. B. (1997). Context and mediators in a Theory of Distributed Associative Memory (TODAM2). Psychological Review, 104, 839-862.

Murdock, B. B., \& Okada, R. (1970). Interresponse times in single-trial free recall. Journal of Experimental Psychology, 86, 263-267.

Neath, I. (1993). Contextual and distinctive processes and the serial position function. Journal of Memory and Language, 32, 820-840.

Nelder, J. A., \& Mead, R. (1965). A simplex method for function minimization. Computer Journal, 7, 308-313.

Postman, L., \& Phillips, L. W. (1965). Short-term temporal changes in free recall. Quarterly Journal of Experimental Psychology, 17, 132-138.

Raaijmakers, J. G. W., \& Shiffrin, R. M. (1981). Search of associative memory. Psychological Review, 88, 93-134.

Sirotin, Y. B., Kimball, D. R., \& Kahana, M. J. (2005). Going beyond a single list: Modeling 
EMPIRICAL AND THEORETICAL LIMITS ON LAG RECENCY IN FREE RECALL22

the effects of prior experience on episodic free recall. Psychonomic Bulletin \& Review, 12, 787-805.

Tan, L., \& Ward, G. (2000). A recency-based account of the primacy effect in free recall. Journal of Experimental Psychology: Learning, Memory, and Cognition, 26, 1589-1625.

Wagenmakers, E.-J., \& Farrell, S. (2004). AIC model selection using Akaike weights. Psychonomic Bulletin \& Review, 11, 192-196.

Zaromb, F. M., Howard, M. W., Dolan, E. D., Sirotin, Y. B., Tully, M., Wingfield, A., et al. (2006). Temporal associations and prior-list intrusions in free recall. Journal of Experimental Psychology: Learning, Memory and Cognition, 32, 792-804. 


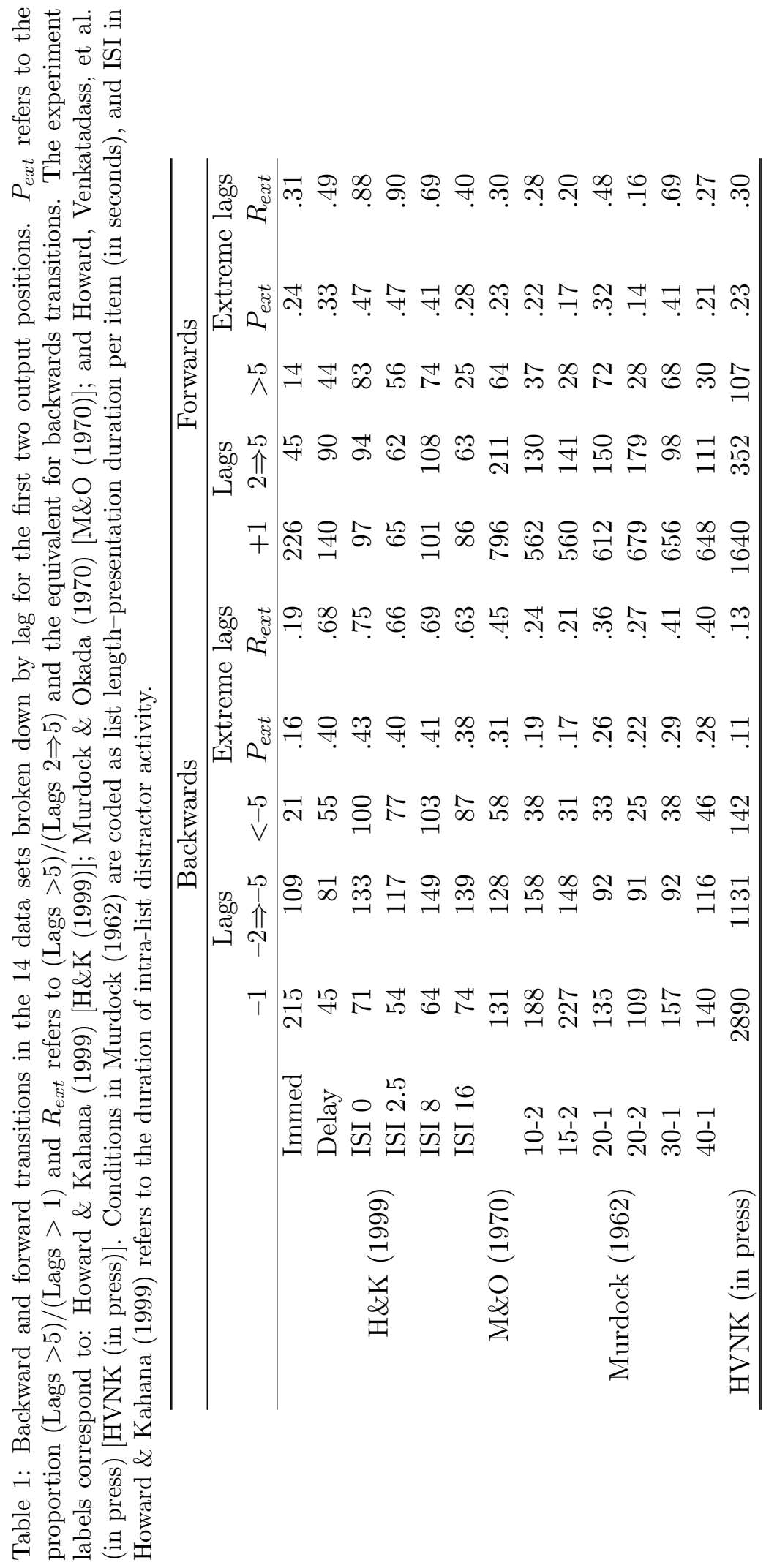




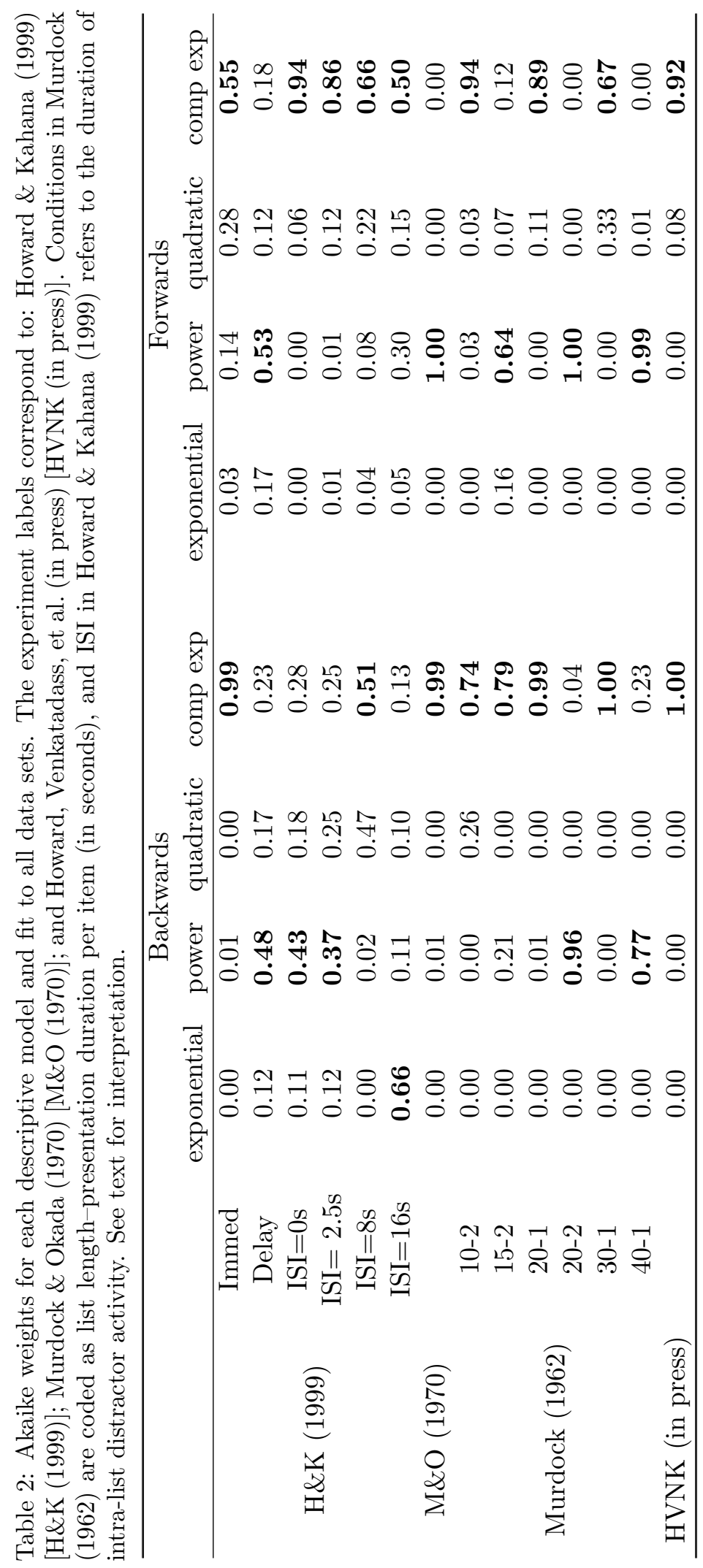


Table 3: Goodness of fit $(-2 \ln L)$ values for two versions of TCM, for each experiment fit. A smaller value indicates a better fit.

\begin{tabular}{ccccc}
\hline & & $\mathrm{TCM}_{\text {pub }}$ & TCM $_{\text {evo }}$ & Critical value $(\alpha=.05)$ \\
\hline & Immed & 3968.6 & 3326.6 & 1253.8 \\
& Delay & 4435.2 & 4413.0 & 983.4 \\
H\&K(1999) & ISI=0 & 5545.5 & 5526.5 & 1248.6 \\
& ISI=2.5 & 3958.5 & 3909.6 & 934.5 \\
& ISI=8 & 5552.0 & 5476.5 & 1302.4 \\
M\&O(1970) & ISI=16 & 4137.6 & 4077.6 & 1035.3 \\
& & 13520.0 & 11772.0 & 2787.2 \\
Murdock(1962) & $10-2$ & 8764.1 & 8149.9 & 2364.5 \\
& $15-2$ & 9731.7 & 8303.8 & 2400.4 \\
& $20-1$ & 10985.0 & 9473.5 & 2320.5 \\
& $20-2$ & 11163.0 & 9585.1 & 2342.0 \\
HVNK (in press) & $40-1$ & 11478.0 & 9274.1 & 2348.1 \\
\hline
\end{tabular}

Table 4: Mean maximum likelihood parameter estimates obtained in fitting each version of TCM to each data set. An explanation of the parameters can be found in the supplementary materials to this article, or Howard \& Kahana (2002a).

\begin{tabular}{cccccc}
\hline & & \multicolumn{3}{c}{$\mathrm{TCM}_{\text {pub }}$} & \multicolumn{3}{c}{ TCM $_{\text {evo }}$} \\
& & $\beta$ & $\tau$ & $\beta$ & $\tau$ \\
\hline & Immed & 0.44 & 0.30 & 0.34 & 0.20 \\
H\&K(1999) & Delay & 0.70 & 0.22 & 0.68 & 0.26 \\
& ISI=0 & 0.56 & 0.29 & 0.59 & 0.42 \\
& ISI=2.5 & 0.61 & 0.26 & 0.59 & 0.32 \\
& ISI=8 & 0.54 & 0.31 & 0.53 & 0.34 \\
M\&O(1970) & ISI=16 & 0.36 & 0.31 & 0.42 & 0.32 \\
& & 0.28 & 0.25 & 0.39 & 0.35 \\
& $10-2$ & 0.34 & 0.29 & 0.27 & 0.23 \\
Murdock(1962) & $15-2$ & 0.39 & 0.27 & 0.24 & 0.15 \\
& $20-1$ & 0.33 & 0.30 & 0.23 & 0.22 \\
& $20-2$ & 0.32 & 0.28 & 0.16 & 0.13 \\
HVNK (in press) & $30-1$ & 0.37 & 0.26 & 0.32 & 0.23 \\
& $40-1$ & 0.38 & 0.21 & 0.29 & 0.20 \\
& & 0.52 & 0.23 & 0.43 & 0.20 \\
\hline
\end{tabular}

